\title{
MUDANÇAS NAS CARACTERÍSTICAS COMPOSICIONAIS DAS ÁGUAS DA LAGOA DE IBIRAQUERA (SC) EM RESPOSTA A DINÂMICA DE ABERTURA E FECHAMENTO DE SUA DESEMBOCADURA
}

\author{
BONETTI, C. ${ }^{1}$; BONETTI, J. ${ }^{1}$ \& BELTRAME, E. ${ }^{2}$ \\ ${ }^{1}$ Laboratório de Oceanografia Costeira - LOC (GCN/UFSC) - cbonetti@cfh.ufsc.br \\ ${ }^{2}$ Laboratório de Camarões Marinhos - LCM (AQI/UFSC)
}

\begin{abstract}
Bonetti, C.; Bonetti, J. \& Beltrame, E. 2005. Changes in water composition of Lagoa de lbiraquera (SC) as a consequence of the opening dynamic of its mouth. Braz. J. Aquat. Sci. Technol. 9(2):39-47. ISSN 1808-7035. The aim of this paper was to hydrochemically characterize Ibiraquera Lagoon (SC) and verify the changes in water column related to the opening dynamics of its mouth. The monthly monitoring of five physical-chemical properties and dissolved inorganic nutrients from march 2003 to april 2004 demonstrated the mixohaline nature of this lagoon, which presents well oxygenated waters, low turbidity and low inorganic dissolved nutrients concentrations. This lagoon is isolated from coastal waters influence in the most part of the year since its sand bar opens only under severe rainy events or by human intervention. On these situations changes in the water level and physical-chemical properties can be observed along all this water body. Among the monitored variables, the most significant alterations were observed in salinity, silica and dissolved oxygen. An increase in salinity and silica values was observed after the sand bar opening in all lagoonal sectors. In contrast, dissolved oxygen concentrations decreased. These changes were associated to the mixture of brackish and marine waters and to nutrient releases from surface bottom sediments to the water column as a consequence of the intensification of circulation processes.
\end{abstract}

Keywords: lagoons, hydrochemistry, sand bar dynamics

\section{INTRODUÇÃO}

Os sistemas lagunares da região sul do Brasil são compostos por corpos d'água salobra que se comunicam com a região costeira adjacente através de canais relativamente estreitos e posicionados perpendicularmente às barreiras arenosas quaternárias. São normalmente sistemas rasos e com alta produtividade biológica, contribuindo de maneira direta para o controle da inundação das planícies costeiras, da estabilidade climática local e do enriquecimento da biodiversidade costeira (Yãnez-Arancibia,1987; Kjerfve, 1994; Esteves, 1998). Todavia, por atuarem também como "filtros", retendo por longos períodos sedimentos inorgânicos, compostos orgânicos e demais materiais carreados para seu interior pelos sistemas fluviais e/ou canais de drenagem, estes ecossistemas são bastante frágeis e susceptíveis a poluição (Knoppers, 1994; Lacerda, 1994).

Devido as baixas profundidades, a lâmina d'água é fortemente influenciada por agentes climatológicos, tais como a precipitação, a evaporação e os ventos, os quais promovem rápidas flutuações em suas características físico-químicas. Embora as marés também exerçam papel importante nos processos hidroquímicos lagunares, sua influência é secundária nos ambientes costeiros sujeitos a micromarés e nas lagunas com comunicação intermitente com o mar. A hidroquímica dos corpos lagunares depende também da fisiografia dos canais de comunicação com o mar e da estabilidade de suas desembocaduras. As lagunas localizadas ao longo de zonas de alta energia de ondas e sujeitas a micromarés, como é o caso da Lagoa de Ibiraquera, normalmente apresentam um canal estreito e raso, periodicamente obstruído por uma barreira arenosa, resultado da deposição de sedimentos marinhos transportados pelas correntes de deriva litorânea e empilhados pela ação de ondas de tempestade. $O$ confinamento induzido pela obstrução parcial ou total da comunicação laguna-oceano restringe a ação das marés e confere ao regime de ventos o papel de principal agente nos processos de circulação interna em corpos onde o aporte fluvial é inexpressivo.

A abertura artificial dessas desembocaduras é uma prática comum nas lagunas costeiras da região sul, já tendo sido relatada na Lagoa do Camacho (SC) por Klein et al. (1998) e Porto Filho \& Elicher (2000). Ela é muitas vezes feita por iniciativa da própria comunidade e visa, entre outros, permitir o escoamento das águas lagunares nas épocas de cheia e promover a entrada de organismos marinhos de interesse econômico. O impacto causado por esta intervenção, no entanto, tem merecido pouca atenção, não se conhecendo ainda a extensão dos danos ecológicos causados pela alteração dos padrões de qualidade de água logo após a abertura da desembocadura. Esteves (1998) cita, 
entre os aspectos negativos da abertura de desembocaduras em curtos intervalos temporais, a instabilidade ecológica decorrente das constantes interrupções dos processos de sucessão das comunidades aquáticas. Mais recentemente, estudos apresentados por Suzuki et al. (1998) e Suzuki et al. (2002) sobre a variabilidade físico-química em lagunas do Rio de Janeiro sujeitas a aberturas artificiais têm quantificado alterações relacionadas à drástica redução do volume de água e suas conseqüências para as comunidades biológicas.

Os objetivos desta pesquisa são: (1) caracterizar hidroquimicamente as águas marginais da Lagoa de Ibiraquera e (2) avaliar a extensão das mudanças físico-químicas provocadas pela alteração dos fluxos lagunares decorrentes da abertura e fechamento do canal de comunicação com o mar.

\section{MATERIAL E MÉTODOS}

\section{Área de Estudo}

A Lagoa de Ibiraquera encontra-se localizada na região sul do estado de Santa Catarina, abrangendo parte dos municípios de Imbituba e Garopaba (SC). A área apresenta-se sob o domínio climático subtropical úmido, com temperatura média anual de $19,63^{\circ} \mathrm{C}$ e precipitação média anual de 1193 mm/ano (SDM/ CLIMERH/EPAGRI, 2001). A distribuição regional dos ventos é controlada pelo sistema de alta pressão do Atlântico Sul, sendo predominante ventos de NE com intensidade média de $12 \mathrm{Km} \cdot \mathrm{h}^{-1}$. Não obstante ventos do quadrante sul, embora menos freqüentes, apresentam maior intensidade.

Esta laguna possui uma lâmina de água de aproximadamente 869 ha e profundidade média inferior a 2 m. Geomorfologicamente pode ser segmentada em quatro setores denominados localmente como Lagoa de Cima, Lagoa do Meio, Lagoa de Baixo e Lagoa do Saco (Figura 1). O canal de ligação entre as águas interiores e as águas marinhas, localizado na Lagoa de Baixo, mantém-se predominantemente fechado, sendo esporadicamente aberto para escoar as águas em época de cheias ou para facilitar a entrada de espécies marinhas de interesse à pesca artesanal. De acordo com a classificação proposta por KJERFVE (1994), esta laguna pode ser classificada como do tipo "shallow choked", possuindo alta relação superfície/volume e restrita troca com o oceano adjacente.

No seu entorno vivem oito comunidades e a atividade pesqueira emprega cerca de 350 pescadores oficialmente cadastrados. As principais espécies capturadas são o camarão-rosa (Farfantepenaeus paulensis e $F$. brasiliensis), o siri-azul (Callinectes sapidus), a tainha e a tainhota (Mugil platanus), segundo dados reunidos pelo projeto "Manejo Integrado da Pesca na Lagoa de Ibiraquera - SC" (UFSC, FNMA, edital 02/ 2002). Apesar de suas características naturais e importância turística, a Lagoa de Ibiraquera tem sido pouco estudada, não existindo séries de dados oceanográficos que subsidiem planos de uso e manejo deste corpo d'água.

\section{Coleta e Análise de Dados}

Este estudo baseia-se em dados físico-químicos adquiridos mensalmente entre abril de 2003 e março de 2004. Foram implementadas 15 estações amostrais distribuídas marginalmente ao longo dos quatro segmentos que compõem o corpo lagunar, conforme posicionamento indicado na Figura 1. Durante as amostragens foram coletados dados in situ de temperatura da água, oxigênio dissolvido e $\mathrm{pH}$, utlizandose uma oxímetro YSI modelo F1055 e um pHmetro marca Goulton modelo AT300, respectivamente. Amostras de água de superfície foram recolhidas para posterior análise em laboratório da salinidade (sonda WTD), turbidez (turbidímetro Orbeco-Hellige) e concentração de nutrientes inorgânicos dissolvidos.

As alíquotas de água destinadas à análise de nutrientes foram mantidas refrigeradas até a chegada no laboratório. Na seqüência elas foram filtradas com filtro Millipore ${ }^{\circledR} \mathrm{HA} 0.45 \mu \mathrm{m}$, divididas em cinco frascos de polietileno de $50 \mathrm{ml}$ e congeladas. Posteriormente foram analisadas quanto à concentração de nitrogênio amoniacal $\left(\mathrm{N}-\mathrm{NH}_{4}\right)$, nitrato $\left(\mathrm{N}-\mathrm{NO}_{3}\right)$, nitrito $\left(\mathrm{N}-\mathrm{NO}_{2}\right)$, fosfato $\left(\mathrm{PO}_{4}\right)$ e silicato $\left(\mathrm{SiO}_{2}\right)$ através da aplicação de técnicas colorimétricas. Análises múltiplas realizadas com soluções padrões entre 0,01 e 5,00 mg. $\mathrm{L}^{-1}$ indicaram os seguintes valores de exatidão do método para cada nutriente analisado: 0,05 mg. $\mathrm{L}^{-1} \mathrm{~N}-\mathrm{NH}_{4} ; 0,30 \mathrm{mg} \cdot \mathrm{L}^{-}$ ${ }^{1} \mathrm{~N}-\mathrm{NO}_{3} ; 0,01 \mathrm{mg} \cdot \mathrm{L}^{-1} \mathrm{~N}-\mathrm{NO}_{2} ; 0,05 \mathrm{mg}^{-\mathrm{L}^{-1}} \mathrm{PO}_{4}$ e 0,10 mg. $\mathrm{L}^{-1} \mathrm{SiO}_{2}$. A partir da somatória dos valores de $\mathrm{N}$ $\mathrm{NH}_{4}, \mathrm{~N}-\mathrm{NO}_{3}$ e N-NO $\mathrm{NO}_{2}$ determinou-se também os valores de NID (nitrogenados inorgânicos dissolvidos). A porcentagem de amônia tóxica $\left(\mathrm{NH}_{3}\right)$ foi calculada segundo Aminot \& Chaussepied (1983). É importante ressaltar que os valores de exatidão dos métodos adotados são restritivos para a caracterização dos teores de nutrientes de águas marinhas, sendo todavia sua aplicação compatível com estudos de detecção de águas costeiras enriquecidas.

\section{Análise Estatística}

Os resultados obtidos ao longo das campanhas de amostragem foram tabulados visando o cálculo dos 


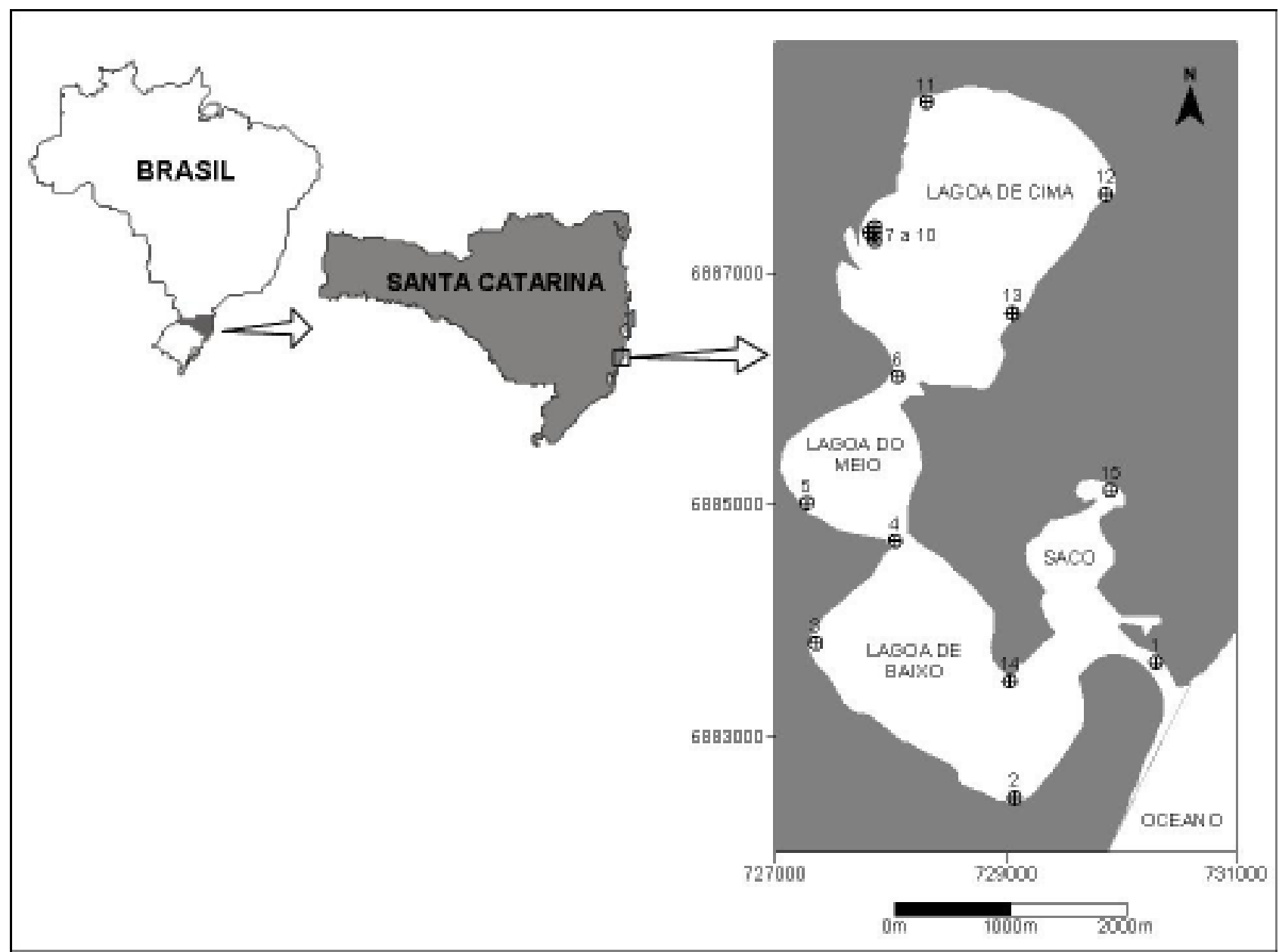

Figura 1 - Localização geográfica da Lagoa de Ibiraquera e posicionamento das estações amostrais.

valores máximo, mínimo, médio e o desvio padrão das propriedades físico-químicas analisadas ao longo dos diferentes segmentos lagunares.

As alterações na qualidade de água da Lagoa de Ibiraquera sob diferentes condições de circulação lagunar foram testadas utilizando-se a técnica da Análise de Variância One-Way (ANOVA). A planilha de dados foi organizada de modo a formar dois grupos temporalmente distintos: $(A)$ dados reunidos entre outubro e dezembro de 2003 sob condição de desembocadura fechada e (B) dados reunidos entre janeiro e março de 2004 sob condição de desembocadura aberta. Os demais meses de monitoramento não foram considerados nesta análise de modo a minimizar as interferências provocadas pelos agentes climatológicos. Nos casos em que a hipótese nula de variância foi rejeitada aplicou-se um teste de comparação múltipla (teste de Tukey - HSD) para identificar quais pares de médias diferiram estatisticamente. Foram consideradas como significantes as diferenças com probabilidade de ocorrência ao acaso menor que $5 \%(p=0.05)$ (Zar, 1998). Os procedimentos estatísticos foram conduzidos utilizando-se os softwares Excel e Statistica for Windows.

\section{RESULTADOS E DISCUSSÃO}

\section{Caracterização Oceanográfica}

Os resultados das campanhas de coleta encontram-se resumidos na Tabela 1, sendo apresentados no formato de valores máximo, mínimo, médio e desvio padrão para cada setor lagunar. O comportamento hidroquímico de cada estação monitorada encontra-se sintetizado na Figura 2. Em termos anuais (abril de 2003 a março de 2004), a Lagoa de Ibiraquera caracterizou-se como um corpo composto por águas mixohalinas (salinidades entre 6,0 e 35,3 PSU), básicas (valores de pH entre 7,01 e 9,15), com temperaturas amenas (média de $23,5 \pm 4,4^{\circ} \mathrm{C}$ ), bem oxigenadas (valores médios acima de $7 \mathrm{mg} \cdot \mathrm{L}^{-1}$ ), com baixa turbidez (valores médios inferiores a 11,0 NTU) e com teores médios de nutrientes inorgânicos dissolvidos dentro dos intervalos esperados para ambientes costeiros não poluídos (Aminot \& Chaussepied, 1983; Day et al., 1987).

Os processos de circulação neste corpo lagunar são dominados pela ação dos ventos, os quais associados à baixa espessura da lâmina d'água e ao reduzi- 
Tabela 1 - Valores médios e desvio padrão, mínimos e máximos das variáveis físico-químicas analisadas nas águas superficiais dos segmentos lagunares da Lagoa da Ibiraquera (período de referência: abril de 2003 a março de 2004).

\begin{tabular}{|c|c|c|c|c|c|c|c|c|c|c|c|c|c|}
\hline SETOR & ESTAÇÃO & DESCRITORES & $\begin{array}{l}\text { SAL. } \\
\text { PSU }\end{array}$ & $\begin{array}{c}\text { TEMP. } \\
{ }^{\circ} \mathrm{C}\end{array}$ & $\mathrm{pH}$ & $\begin{array}{c}\text { TURB } \\
\text { NTU }\end{array}$ & $\begin{array}{c}\mathrm{O}_{2} \\
\mathrm{mg} \cdot \mathrm{L}^{-1}\end{array}$ & $\begin{array}{l}\mathrm{N}-\mathrm{NH} 4 \\
\mathrm{mg} \cdot \mathrm{L}^{-1}\end{array}$ & $\begin{array}{l}\mathrm{N}-\mathrm{NO} 3 \\
\mathrm{mg} \cdot \mathrm{L}^{-1}\end{array}$ & $\begin{array}{l}\mathrm{N}-\mathrm{NO} 2 \\
\mathrm{mg} \cdot \mathrm{L}^{-1}\end{array}$ & $\begin{array}{c}\text { DIN } \\
\text { mg.L }\end{array}$ & $\begin{array}{l}\mathrm{SiO} 2 \\
\mathrm{mg} \cdot \mathrm{L}^{-1}\end{array}$ & $\begin{array}{c}\mathrm{PO} 4 \\
\text { mg. } \mathrm{L}^{-1}\end{array}$ \\
\hline \multirow{4}{*}{ 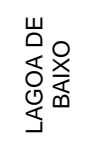 } & \multirow{4}{*}{$\begin{array}{l}\vec{J} \\
\dot{m} \\
\dot{N} \\
\dot{H}\end{array}$} & valor médio & 22,0 & 23,4 & 8,28 & 7,8 & 8,12 & 0,00 & 0,20 & 0,01 & 0,21 & 1,28 & 0,06 \\
\hline & & desvio padrão & 7,4 & 4,2 & 0,23 & 9,9 & 1,36 & 0,01 & 0,31 & 0,01 & 0,31 & 0,52 & 0,07 \\
\hline & & valor mínimo & 7,4 & 16,4 & 7,40 & 1,6 & 4,13 & 0,00 & 0,00 & 0,00 & 0,00 & 0,42 & 0,00 \\
\hline & & valor máximo & 35,3 & 32,7 & 8,65 & 55,0 & 11,20 & 0,03 & 1,26 & 0,02 & 1,26 & 2,41 & 0,22 \\
\hline \multirow{4}{*}{ 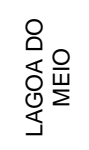 } & \multirow{4}{*}{$\begin{array}{l}0 \\
\dot{n} \\
\ddot{\nabla}\end{array}$} & valor médio & 21,1 & 23,4 & 8,33 & 5,9 & 7,76 & 0,01 & 0,24 & 0,01 & 0,26 & 1,48 & 0,07 \\
\hline & & desvio padrão & 5,9 & 3,9 & 0,26 & 2,7 & 1,25 & 0,05 & 0,30 & 0,01 & 0,31 & 0,68 & 0,12 \\
\hline & & valor mínimo & 12,0 & 16,5 & 7,90 & 2,7 & 5,13 & 0,00 & 0,00 & 0,00 & 0,01 & 0,01 & 0,00 \\
\hline & & valor máximo & 30,9 & 32,3 & 9,15 & 13,5 & 9,60 & 0,24 & 1,32 & 0,02 & 1,33 & 2,81 & 0,47 \\
\hline \multirow{4}{*}{ 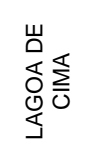 } & \multirow{4}{*}{ 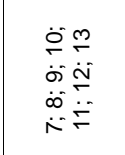 } & valor médio & 17,3 & 23,7 & 8,08 & 6,5 & 7,16 & 0,19 & 0,21 & 0,01 & 0,40 & 1,36 & 0,08 \\
\hline & & desvio padrão & 4,8 & 4,4 & 0,40 & 4,5 & 1,16 & 0,28 & 0,24 & 0,01 & 0,37 & 0,86 & 0,10 \\
\hline & & valor mínimo & 6,6 & 16,2 & 7,01 & 1,0 & 4,78 & 0,00 & 0,00 & 0,00 & 0,00 & 0,00 & 0,00 \\
\hline & & valor máximo & 25,2 & 33,8 & 9,02 & 21,3 & 9,10 & 1,15 & 0,91 & 0,02 & 1,51 & 3,04 & 0,47 \\
\hline \multirow{4}{*}{ 足 } & \multirow{4}{*}{$\stackrel{2}{\sim}$} & valor médio & 15,3 & 23,7 & 8,33 & 8,4 & 7,86 & 0,02 & 0,44 & 0,01 & 0,47 & 1,22 & 0,03 \\
\hline & & desvio padrão & 5,3 & 4,4 & 0,38 & 5,5 & 1,73 & 0,03 & 0,34 & 0,01 & 0,34 & 0,77 & 0,06 \\
\hline & & valor mínimo & 6,9 & 16,2 & 7,60 & 3,8 & 5,15 & 0,00 & 0,00 & 0,00 & 0,09 & 0,22 & 0,00 \\
\hline & & valor máximo & 22,3 & 33,8 & 8,78 & 18,6 & 10,60 & 0,08 & 1,26 & 0,02 & 1,31 & 2,28 & 0,19 \\
\hline
\end{tabular}

do aporte de material orgânico particulado levam a uma condição ideal de oxigenação das águas. Conforme exposto na Figura 2, a maioria das estações apresentou valores médios de oxigênio dissolvido entre 6,0 e $9,0 \mathrm{mg} \cdot \mathrm{L}^{-1}$, sendo que as maiores flutuações foram registradas nas estações 2,3 e 15 . No caso das estações 2 e 15 foram encontrados valores máximos acima de $10 \mathrm{mg} \cdot \mathrm{L}^{-1}$, o que pode estar indicando momentos de maior produtividade primária. Como ambos os trechos estão sujeitos ao aporte de drenagem continental, ainda que este seja pouco expressivo, pode-se supor que esteja ocorrendo um favorecimento das populações fitoplanctônicas em reposta ao aporte de nutrientes. Estas estações também apresentaram valores mais baixos de salinidade (média de 20,8 \pm 7,8 PSU para a estação 2 e 15,7 \pm 5,2 PSU para a estação 15) e picos de maior turbidez (média de 11,0 \pm 17,9 NTU para a estação 2 e 8,2 \pm 5,1 NTU para a estação 15). Já a estação 3, embora em termos médios tenha se comportado como as demais em relação a todas as propriedades monitoradas, em fevereiro de 2004 ela apresentou uma brusca redução dos teores de oxigênio dissolvido, registrando-se um valor de 4,3 mg. $\mathrm{L}^{-1}$. Embora este valor ainda indique condição de oxigenação satisfatória, ele encontra-se bem abaixo da média lagunar observada.

Analisando-se individualmente cada setor lagunar, encontra-se um gradiente decrescente de influência marinha no sentido Lagoa de Baixo - Lagoa de Cima, o que se justifica pelo maior distanciamento deste último setor da desembocadura marinha. Como não existe um aporte fluvial bem definido ao longo de toda a laguna, a influência de águas doces é pouco expressiva, ocorrendo de forma dispersa a partir de drenagens pluviais. Conforme já assinalado acima, destaca-se neste sentido a contribuição de um pequeno canal que deságua na porção sul da Lagoa de Cima e que se comunica com uma pequena lagoa de água doce localizada à retaguarda do campo de dunas local. A contribuição das águas provenientes deste canal justifica as alterações nos valores de salinidade, oxigênio dissolvido e, sobretudo, de turbidez observados na estação 2 .

Com base na distribuição dos valores médios anuais de salinidade podemos dividir esta laguna em dois segmentos:

- setor polihalino: com salinidades entre 18 e 30 PSU, abrangendo as estações localizadas na Lagoa de Baixo $(1,2,3,14)$ e a na Lagoa do Meio (4, 5 e 6); - setor mesohalino: com salinidades entre 5 e 18 PSU, abrangendo as estações localizadas na Lagoa de Cima $(7,8,9,10,11,12,13)$ e no Saco (15).

Apesar da significativa diferenciação destes segmentos lagunares quanto à salinidade, não foram observadas correspondências entre esta divisão e a distribuição espacial das demais propriedades fisico-químicas analisadas.

Em relação à distribuição espaço-temporal dos nutrientes inorgânicos dissolvidos, não houve uma diferenciação significativa dos segmentos lagunares quanto aos valores médios observados (Figura 3), com exceção do amônio. Este elemento foi encontrado em concentrações mais altas na Lagoa de Cima (valor médio anual de $0,19 \mathrm{mg}^{\mathrm{L}} \mathrm{L}^{-1}$ ), indicando que neste trecho pode estar ocorrendo um aporte preferencial de compostos 


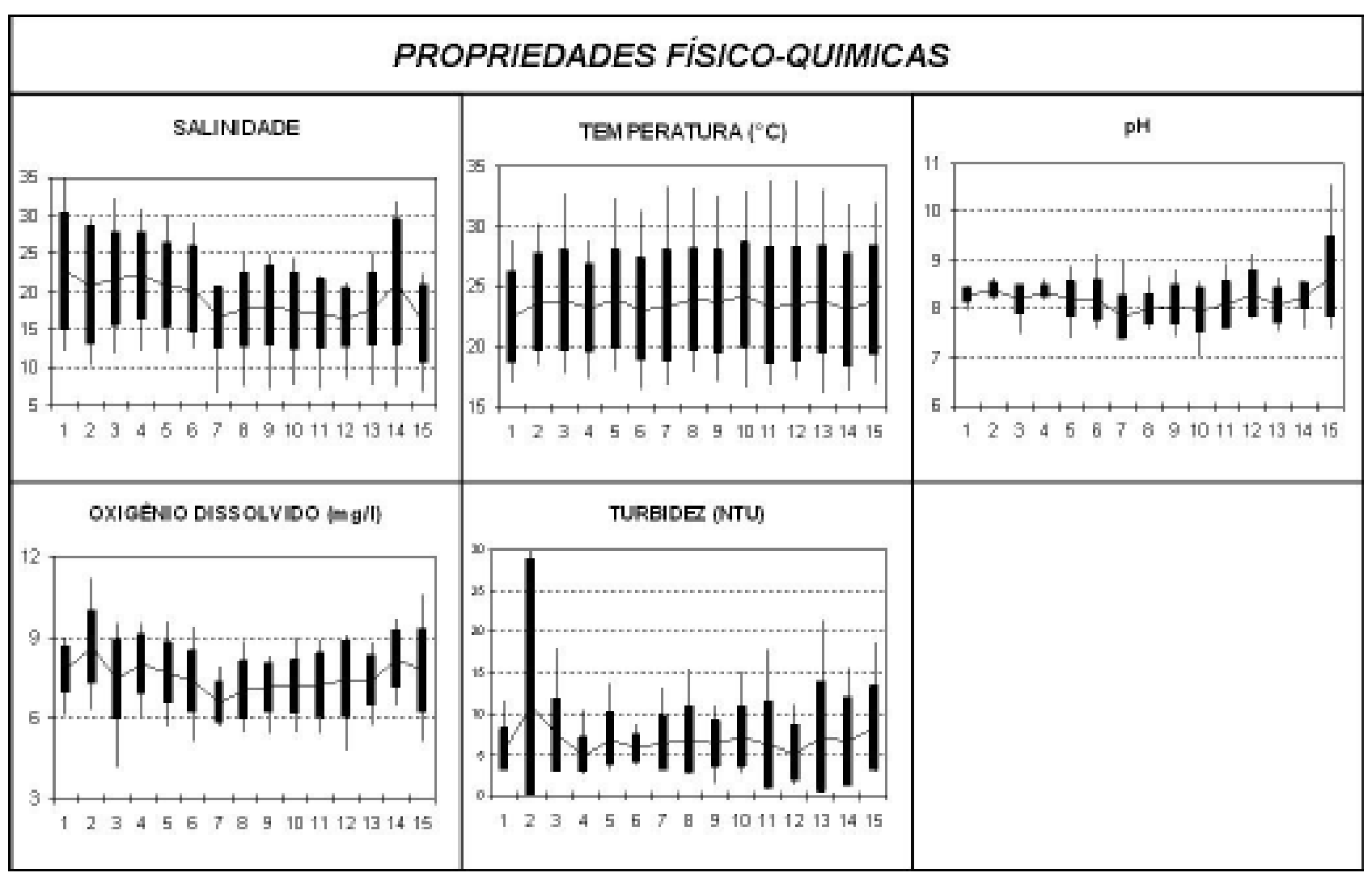

Figura 2 - Distribuição espacial dos valores média \pm desvio padrão, máximo e mínimo das propriedades físico-químicas monitoradas ao longo das 15 estações de amostragem (período de referência: abril de 2003 a março de 2004).

orgânicos. Não foram verificados, no entanto, aumento dos teores de nitrato, nitrito, ortofosfato e sílica neste setor. Segundo Baumgarten \& Niencheski (1998) o predomínio de amônio em relação às demais formas nitrogenadas pode estar indicando a remineralização da matéria orgânica em condições de baixa oxigenação. Embora tal hipótese esteja em discordância com os valores de oxigênio dissolvido medidos no momento das coletas, é possível que ao longo do ciclo diário ocorram períodos noturnos de hipoxia (processo comum em áreas onde a taxa fotossintética é elevada). As concentrações de nitrato variaram entre "não determinada" e 1,32 mg. $\mathrm{L}^{-1}$, este último ocorrendo na Lagoa do Meio (estação 6) em dezembro de 2003. Em termos médios, os valores mensais mais altos foram observados no segmento do Saco, obtendo-se concentrações de 1,23 mg.L ${ }^{-1} \mathrm{~N}-\mathrm{NO}_{3}$ em maio de 2003 e de $1,26 \mathrm{mg} \cdot \mathrm{L}^{-1} \mathrm{~N}-\mathrm{NO}_{3}$ no mês de janeiro de 2004. O nitrito apresentou-se sempre inferior a $0,02 \mathrm{mg} \cdot \mathrm{L}^{-1}$ ao longo de todo o corpo lagunar. Os valores de NID foram inferiores a $1,51 \mathrm{mg}^{-L^{-1}} \mathrm{em}$ todas as coletas, sendo as maiores concentrações encontradas na Lagoa de Cima.

Os teores de fosfato variaram entre traço e 0,47 $\mathrm{mg} . \mathrm{L}^{-1}$, encontrando-se os maiores valores mensais médios na Lagoa de Cima nos meses de abril e maio de $2003\left(0,22 \mathrm{mg} \cdot \mathrm{L}^{-1} \mathrm{PO}_{4}\right)$ e na Lagoa de Baixo em março de $2004\left(0,23 \mathrm{mg} \cdot \mathrm{L}^{-1} \mathrm{PO}_{4}\right)$. A distribuição dos teores de silicato variou entre $0,01 \mathrm{mg} \cdot \mathrm{L}^{-1}$ e 3,63 mg. $\mathrm{L}^{-}$ ${ }^{1}$, sendo a média anual mais alta encontrada na Lagoa do Meio (1,66 mg. L-1 $\left.\mathrm{SiO}_{2}\right)$. A Lagoa de Cima e a Lagoa de Baixo apresentaram valores médios anuais muito semelhantes $\left(1,41\right.$ e $1,44 \mathrm{mg} . \mathrm{L}^{-1} \mathrm{SiO}_{2}$, respectivamente). Os baixos valores de silicatos podem ser explicados pela inexistência de fontes significativas e/ou pela atuação de um eficiente sistema interno de absorção relacionado à presença de organismos consumidores deste nutriente, sobretudo diatomáceas (Carmouze, 1994).

Comparando-se os teores médios de nutrientes inorgânicos dissolvidos encontrados entre abril de 2003 e março de 2004 em Ibiraquera com resultados apresentados para outros corpos lagunares do Brasil, pode se dizer que:

- quanto aos nitrogenados, o corpo lagunar estudado possui concentrações de amônio inferiores aos da Lagoa da Conceição (Fonseca et al., 2002) e aos da Lagoa dos Patos (Baumgarten \& Niencheski, 1998; Windom et al., 1999). Concentrações médias dentro da mesma ordem de grandeza foram encontradas na Lagoa de Araruama por Souza et al. (2003) e na Lagoa de Imboassica por Kozlowsky-Suzuki \& Bozelli (2002). Os teores de nitrato e nitrito foram superiores aos da 


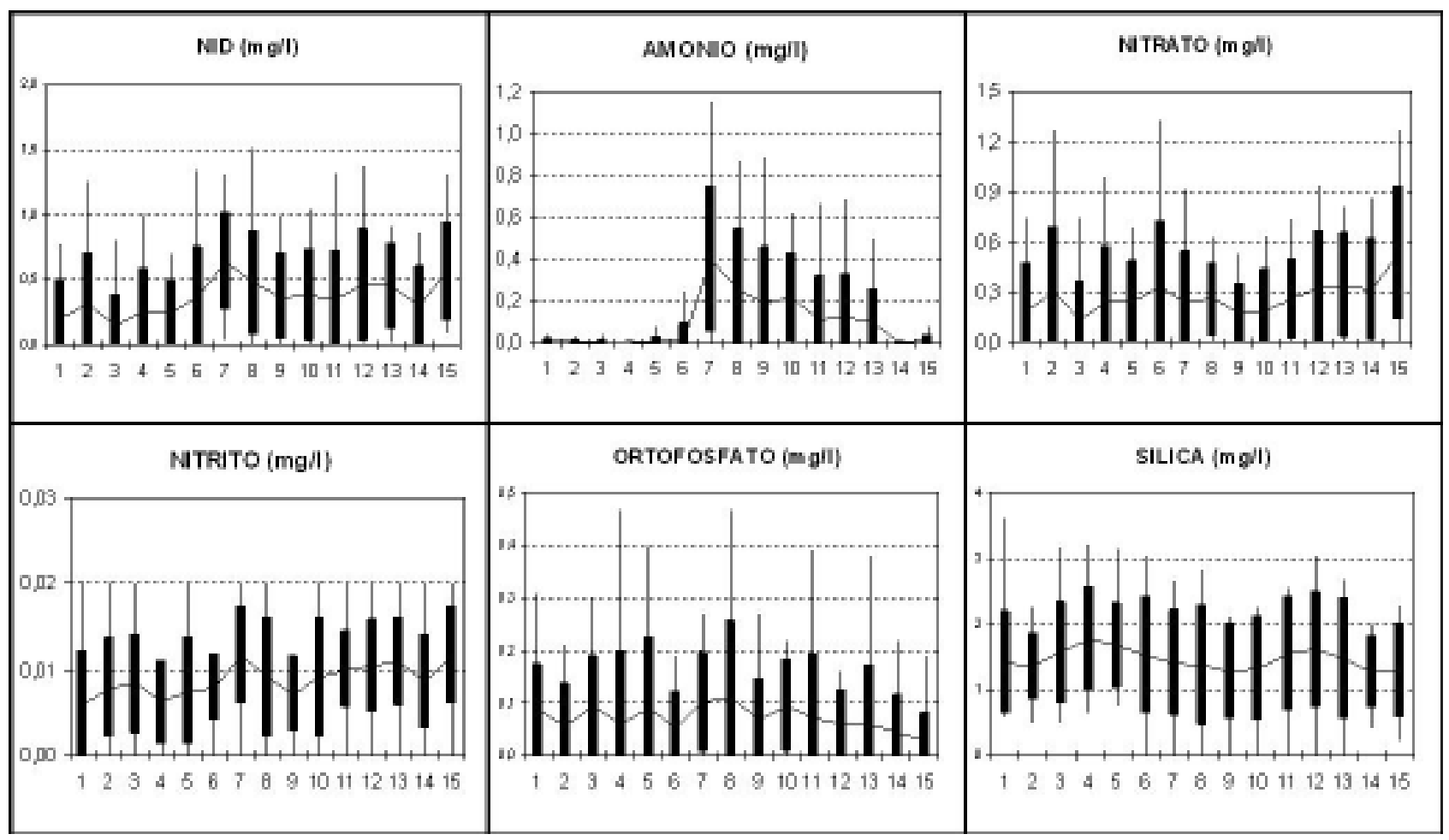

Figura 3 - Distribuição dos valores média \pm desvio padrão, máximo e mínimo dos sais nutrientes monitorados ao longo das 15 estações de amostragem (período de referência: abril de 2003 a março de 2004).

Lagoa da Conceição e inferiores aos da Lagoa dos Patos.

- quanto aos fosfatos, os valores encontrados em Ibiraquera foram semelhantes aos da Lagoa da Conceição e de Araruama, superiores aos de Imboassica e inferiores aos da Lagoa dos Patos.

Mudanças hidroquímicas após a abertura da desembocadura lagunar

A dinâmica de abertura e fechamento do canal de comunicação desta laguna com o mar é a principal responsável pelas variações observadas nas propriedades físico-químicas e na altura da coluna d'água. Entre dezembro e janeiro o nível do corpo lagunar baixou cerca de 1,05 m, conforme registros obtidos a partir de uma régua maregráfica instalada na margem do canal de comunicação entre a Lagoa de Cima e a Lagoa do Meio. A comparação do comportamento da coluna d'água monitorado três meses antes do rompimento da barra que obstrui a desembocadura (de outubro a dezembro de 2003) e três meses após este evento (de janeiro a março de 2004) demonstrou que todos os setores lagunares respondem a esta alteração do fluxo, notando-se um gradiente decrescente de amplitude das mudanças no sentido Lagoa de Baixo - Lagoa de Cima (Tabela 2; Figura 4). Segundo os resultados da análise de variância, alterações significativas para á menor e igual a 0.05 foram observadas em relação à salinidade, oxigênio dissolvido e teores de silicato em todos os segmentos lagunares (Tabela 3).

$\mathrm{Na}$ Lagoa de Baixo e Lagoa do Saco, representados pelas estações 1, 2, 3, 14 e 15, a salinidade média antes da abertura da barra era de 13,06 PSU (águas mesohalinas) e após a abertura passou para 27,53 PSU (águas polihalinas). Esta mudança reflete a mistura das águas interiores com as águas marinhas, a qual, por sua vez, parece ter determinado a redução dos valores de oxigênio dissolvido. Comportamento semelhante foi observado também na Lagoa do Meio (estações 4, 5 e 6). Por fim, na Lagoa de Cima foram observadas mudanças significativas nos valores de salinidade (12,60 para 20,09 PSU), oxigênio dissolvido (de 7,40 para $5,81 \mathrm{mg} \cdot \mathrm{L}^{-1}$ ) , sílica (de 0,85 para 1,89 mg. $\mathrm{L}^{-1}$ ) e turbidez (de 9,86 para 3,70 mg. $\mathrm{L}^{-1}$ ).

Embora não tenham sido detectadas mudanças estatisticamente significativas em relação ao comportamento dos nitrogenados, notou-se em janeiro uma redução das concentrações na Lagoa do Meio e o aumento destas na Lagoa de Baixo e na Lagoa do Saco, sendo que a Lagoa de Cima permaneceu com concentrações semelhantes a do período em que a barra encontrava-se fechada. Já em fevereiro houve uma redução das concentrações na Lagoa de Cima e na Lagoa do Meio, mantendo-se ainda altas na Lagoa de Baixo e na da Lagoa do Saco. Por fim, no mês de março, todos os segmentos lagunares apresentaram concentrações mais baixas que as registradas anteriormente. Este 

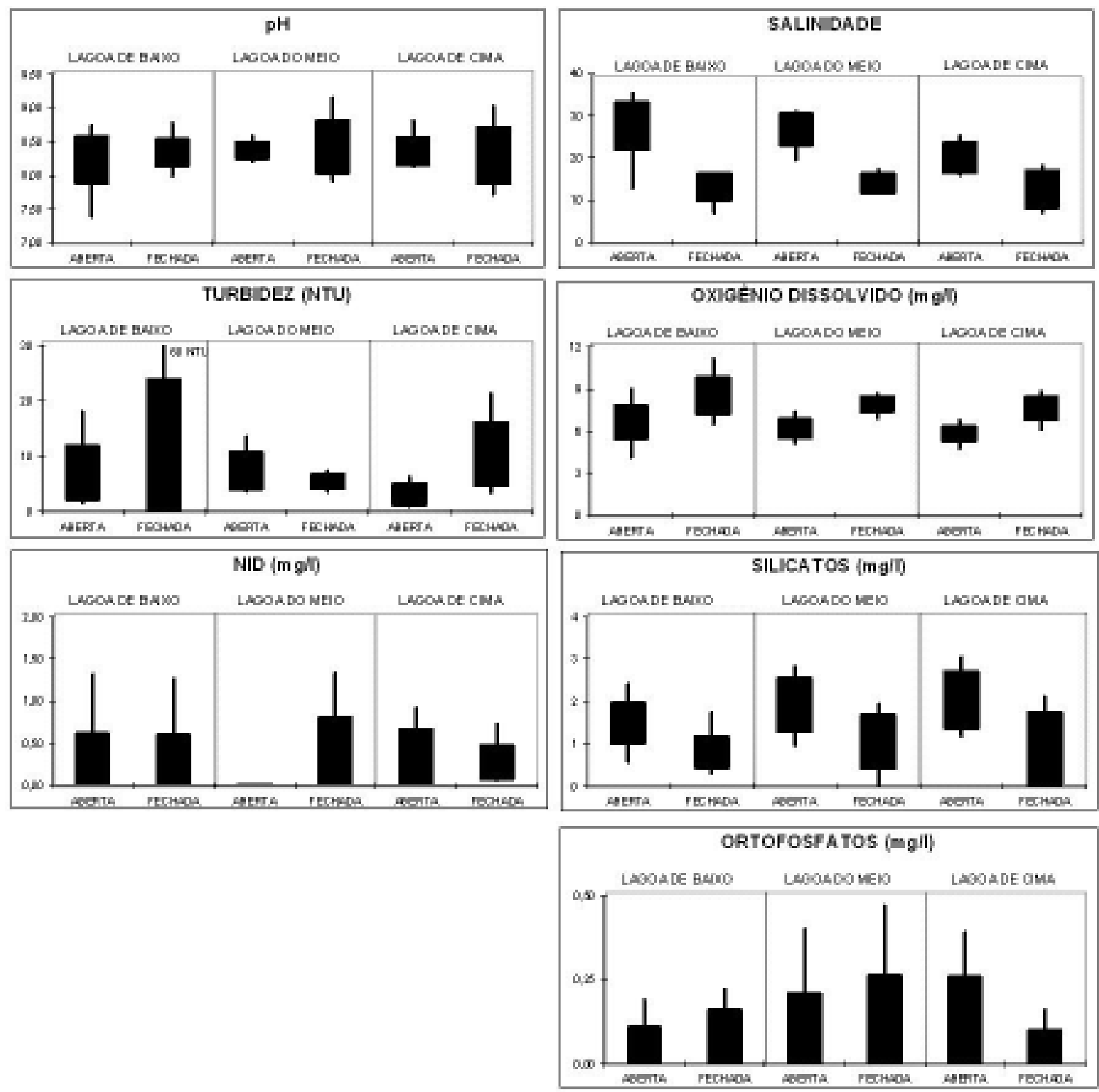

Figura 4 - Distribuição dos valores média \pm desvio padrão, máximo e mínimo das variáveis monitoradas nos segmentos lagunares antes e após a abertura da desembocadura (período de referência: outubro de 2003 a março de 2004).

comportamento, embora precise ser melhor investigado, pode ser um indicativo do tempo escoamento das águas mixohalinas ao longo do eixo longitudinal após a abertura da desembocadura. A exportação de nutrientes dos sistemas lagunares para as águas costeiras adjacentes em condições semelhantes já foi relatado anteriormente por Suzuki et al. 2002 para a Lagoa de Iquipari (RJ).

$\mathrm{O}$ aumento das concentrações de silicatos ao longo de todo o corpo lagunar após a abertura da desembocadura, embora pareça contraditório com a natureza predominante deste elemento (associado à drenagem continental) pode ser explicado pela intensifica- ção dos processos de remobilização dos sedimentos de fundo à medida que houve um aumento dos processos físicos internos desencadeados pela entrada de águas marinhas e pela redução do nível da laguna.

\section{CONSIDERAÇÕES FINAIS}

A Lagoa de Ibiraquera apresenta características hidroquímicas típicas de um ecossistema transicional sem a influência de aporte continental significativo. Neste sentido, não existe um gradiente espacial de variação das propriedades físico-químicas bem definido, sendo 
Bonetti et al.: Hidroquímica da lagoa de Ibiraquera.

Tabela 2 - Valores médios das variáveis analisadas nas águas superficiais dos segmentos lagunares da Lagoa da lbiraquera antes e após a abertura da desembocadura (período de referência: outubro de 2003 a março de 2004).

\begin{tabular}{|c|c|c|c|c|c|c|c|c|c|c|c|}
\hline $\begin{array}{l}\text { SEGMENTO } \\
\text { LAGUNAR }\end{array}$ & $\begin{array}{l}\text { SITUAÇÃO } \\
\text { DA BARRA }\end{array}$ & $\begin{array}{c}\text { TURB. } \\
\text { NTU }\end{array}$ & $\begin{array}{l}\text { SAL. } \\
\text { PSU }\end{array}$ & $\mathrm{pH}$ & $\begin{array}{c}\mathbf{O}_{\mathbf{2}} \\
\mathrm{mg} \cdot \mathrm{L}^{-1}\end{array}$ & $\begin{array}{l}\mathbf{N}-\mathbf{N H}_{\mathbf{4}} \\
\mathrm{mg} \cdot \mathrm{L}^{-1}\end{array}$ & $\begin{array}{l}\mathrm{N}-\mathrm{NO}_{3} \\
\mathrm{mg} \cdot \mathrm{L}^{-1}\end{array}$ & $\begin{array}{c}\mathrm{N}-\mathrm{NO}_{2} \\
\mathrm{mg} \cdot \mathrm{L}^{-1}\end{array}$ & $\begin{array}{c}\text { NID } \\
\text { mg.L- }{ }^{1}\end{array}$ & $\begin{array}{c}\mathrm{SiO}_{2} \\
\mathrm{mg} \cdot \mathrm{L}^{-1}\end{array}$ & $\begin{array}{c}\mathrm{PO}_{4} \\
\mathrm{mg.} \mathrm{L}^{-1}\end{array}$ \\
\hline \multirow{2}{*}{$\begin{array}{l}\text { LAGOA DE BAIXO } \\
\text { E SACO }\end{array}$} & FECHADA & 10,73 & 13,06 & 8,33 & 8,49 & 0,00 & 0,26 & 0,01 & 0,26 & 0,79 & 0,08 \\
\hline & ABERTA & 6,97 & 27,53 & 8,23 & 6,63 & 0,01 & 0,20 & 0,01 & 0,21 & 1,48 & 0,04 \\
\hline \multirow{2}{*}{ LAGOA DO MEIO } & FECHADA & 5,50 & 14,02 & 8,42 & 7,92 & 0,00 & 0,39 & 0,01 & 0,40 & 1,04 & 0,10 \\
\hline & ABERTA & 7,38 & 26,79 & 8,37 & 6,18 & 0,00 & 0,00 & 0,01 & 0,01 & 1,91 & 0,08 \\
\hline \multirow{2}{*}{ LAGOA DE CIMA } & FECHADA & 9,86 & 12,60 & 8,24 & 7,40 & 0,00 & 0,25 & 0,01 & 0,26 & 0,85 & 0,06 \\
\hline & ABERTA & 3,70 & 20,03 & 8,23 & 5,81 & 0,13 & 0,20 & 0,01 & 0,34 & 1,89 & 0,12 \\
\hline
\end{tabular}

Tabela 3 - Resultado da Análise de Variância entre o comportamento hidroquímico dos segmentos lagunares antes e após a abertura da desembocadura. Os valores sublinhados indicam as variáveis que apresentaram diferenças significativas $($ valores de á 0,05$)$.

\begin{tabular}{|c|c|c|c|c|c|c|c|c|c|c|c|}
\hline \multirow{2}{*}{$\begin{array}{l}\text { SEGMENTO } \\
\text { LAGUNAR }\end{array}$} & \multirow{2}{*}{$\lambda$} & \multirow{2}{*}{$\alpha$} & \multicolumn{9}{|c|}{ Teste de Tukey HSD - valores de probabilidade } \\
\hline & & & $\mathrm{pH}$ & TURB. & SAL. & $\mathrm{O}_{2}$ & N-NH4 & N-NO3 & DIN & $\mathrm{SiO2}$ & PO4 \\
\hline LAGOA DE BAIXO & 0,170 & $\underline{0,005}$ & 0,325 & 0,306 & $\underline{0,000}$ & $\underline{0,000}$ & 0,099 & 0,650 & 0,692 & $\underline{0,000}$ & 0,136 \\
\hline LAGOA DO MEIO & 0,098 & $\underline{0,000}$ & 0,739 & 0,130 & $\underline{0,000}$ & $\underline{0,000}$ & 0,337 & $\underline{0,015}$ & $\underline{0,016}$ & $\underline{0,020}$ & 0,758 \\
\hline LAGOA DE CIMA & 0,088 & $\underline{0,000}$ & 0,960 & $\underline{0,000}$ & $\underline{0,000}$ & $\underline{0,000}$ & 0,192 & 0,479 & 0,385 & $\underline{0,006}$ & 0,093 \\
\hline
\end{tabular}

a heterogeneidade encontrada entre os segmentos lagunares função, sobretudo, do distanciamento da desembocadura marinha e da configuração fisiográfica destes setores. Este último fator condiciona a ação dos ventos nos processos de mistura da coluna d'água e na remobilização de compostos depositados nos sedimentos superficiais de fundo.

Considerando os aspectos físico-químicos e a disponibilidade de sais nutrientes na coluna d'água, a abertura esporádica da desembocadura lagunar de Ibiraquera promove mudanças significativas em todos os setores lagunares. As variáveis que melhor responderam as alterações nos processos de mistura foram a salinidade, o oxigênio dissolvido e os teores de silicato. Vale ressaltar, no entanto, que eventuais mudanças nos teores de nitrogenados e fosfatos podem não ter sido detectadas devido a baixa resolução do método analítico adotado. A análise mensal dos resultados após a abertura da barra leva a crer que as mudanças mais drásticas ocorrem ao longo do primeiro mês.

Com base nos resultados obtidos sugere-se, como continuidade deste estudo, que sejam realizadas amostragens temporais menos espaçadas logo após a abertura da desembocadura, de modo a se avaliar com maior precisão o tempo necessário para que este ecossistema retorne a sua condição de equilíbrio. Seria interessante ainda associar aos dados abióticos, à resposta das comunidades fitoplanctônicas, visando a modelagem ecológica do fenômeno. Acredita-se que a disponibilização destas informações seja de fundamental interesse a elaboração de planos de manejo deste ecossistema que visem à otimização das atividades pesqueiras, assim como a manutenção da qualidade da água desta laguna.

\section{AGRADECIMENTOS}

Os autores agradecem o apoio dos alunos do curso de Engenharia da Aqüicultura e do Programa de Pós-Graduação em Geografia da UFSC que participaram das campanhas de coleta de dados. Em especial, merece nosso reconhecimento o auxílio de Diego Carvalho Sander, responsável técnico pelo setor de qualidade da água do Laboratório de Oceanografia Costeira e do Laboratório de Camarões Marinhos.

\section{REFERENCIAS}

Aminot, A. \& Chaussepied, M. 1983. Manuel des Analyses Chimiques en Milieu Marin. CNEXO, Brest, $395 \mathrm{p}$.

Baumgarten, M.G. Z. \& Niencheski, L. F. H. 1998. Avaliação da qualidade hidroquímica da área portuária da cidade do Rio Grande - RS. Documentos Técnicos Oceanografia, 9: 1-66.

Carmouze, J.P. 1994. O metabolismo dos ecossistemas aquáticos: fundamentos teóricos, métodos de estudo e análises químicas. Editora Edgard Blücher/ Fapesp, São Paulo, 267 p. 
Esteves, F.A. 1998. Ecologia das Lagoas Costeiras do Parque Nacional da Restinga de Jurubatiba e do Município de Macaé (RJ). UFRJ/ NUPEM, Rio de Janeiro, $442 \mathrm{p}$.

Fonseca, A; Braga, E. \& Eichler, B.B. 2002. Distribuição espacial dos nutrientes inorgânicos dissolvidos e da biomassa fitoplanctônica no sistema pelágico da Lagoa da Conceição, Santa Catarina, Brasil. Atlântica, Rio Grande, 24(2): 69-83, 2002.

Kjerfve, B. 1994. Coastal Lagoon Processes, p. 1-8. In: B. Kjerfve (Ed.), Coastal Lagoon Processes. Elsevier Oceanography Series no. 60, Amsterdam, $577 \mathrm{p}$.

Klein, A.H.F.; Schettini, C.A.F.; Carvalho, J.L.B.\& Diehl, F.P. 1998. Implications of inlet stabilization on the Camacho Lagoon, Southern Brazil. Journal of Coastal Research, SI(26):150-155.

Knoppers, B. 1994. Aquatic primary production., p. 221241. In: B. Kjerfve (Ed.), Coastal Lagoon Processes. Elsevier Oceanography Series no. 60, Amsterdam, $577 \mathrm{p}$.

Kozlowsky-Suzuki, B. \& Bozelli, R.L. 2002. Experimental evidence of the effect of nutrient enrichment on the zooplankton in a brazilian coastal lagoon. Braz. J. Biol., 62 (4B): 835-846.

Lacerda, L. D. 1994. Biogeochemistry of heavy metals in coastal lagoons, p. 221-241. In: B. Kjerfve (Ed.), Coastal Lagoon Processes. Elsevier Oceanography Series no. 60, Amsterdam, $577 \mathrm{p}$.

Porto Filho, E., Elicher, M.J. 2000. A sustentabilidade da exploração e manejo dos recursos naturais nas lagoas do Camacho e Garopaba do Sul, Santa
Catarina, Brasil: implicações da abertura artificial da barra do Camacho. p. 1-8. In: Anais do $8^{\circ}$ Encontro de Geógrafos da América Latina, Chile.

SDM/CLIMERH/EPAGRI, 2001. Dados climatológicos das estações meteorológicas da bacia RH9.

Souza, M. F. L.; Kjerfve, B.; Knoppers, B.; Souza, W. F. L. \& Damasceno, R. N. 2003. Nutrient budgets and trophic state in a hypersaline coastal lagoon: Lagoa de Araruama, Brazil. Estuarine, Coastal and Shelf Science 57 (2003): 843-858.

Suzuki, M.S.; Figueiredo, R.O.; Castro, S.C.; Silva, C. F. \& Pereira, E.A.; Silva, J.A. \& Aragon, G.T. 2002. Sand Bar Opening in a Coastal Lagoon (Iquipari) in the Northern Region of Rio de Janeiro State: Hydrological and Hydrochemical Changes. Braz. J. Biol., 62(1): 51-62.

Suzuki, M.S.; Ovalle, A.R.C. \& Pereira, E.A. 1998. Effects of sand bar openings on some limnological variables in a hypertrophic tropical coastal lagoon, Brazil. Hydrobiologia, 368: 111-122.

Windom, H. L.; Niencheski, L. F. \& Smith Jr., R.G. 1999. Biogeochemistry of nutrients and trace metals in the estuarine region of the Patos Lagoon (Brazil). Estuarine, Coastal and Shelf Science 48, 113-123.

Yãnez-Arancibia, A. 1987. Lagunas costeras y estuarios: cronolia, criterios, y conceptos para una clasificación ecológica de sistemas costeros. Revista de la Sociedad Mexicana de Historia Natural, 39: 35-54.

Zar, J. H. 1998. Biostatistical Analysis, 4th Ed. PrenticeHall, UK, 660 p. 